\title{
Virtual Scanning: A New Medical Paradigm?
}

\section{Graham Wilfred Ewing*}

Montague Healthcare, Mulberry House, 6 Vine Farm Close, Cotgrave, Nottingham, UK

\begin{abstract}
The medical profession adopts new medical technologies which are deemed necessary to diagnose and treat disease. The current range of technologies allows the clinician to make diagnostic conclusions although the scope, limitations and costs of such diagnostic techniques are often ignored. The aim of this review article is to consider the techniques currently used to diagnose health and to highlight a novel and innovative way of screening the health of the population using a non-invasive cognitive technique.

In recent years there has been the emergence of many new medical technologies which adopt vague principles yet instead of reducing the cost of diagnosing disease, each new test adds to the complexity and cost of the healthcare system. In addition, each new test invariably introduces limitations/errors which can influence the test outcomes eg. (i) the genetic profile associated with diabetes differs according to the racial origins of each patient, (ii) diseases are often multi-systemic, therefore the association of one biomarker as a diagnostic indication may be inherently flawed, (iii) genetic screening is not fully reproducible due to profiling errors and (iv) the significance of phenotype is often overlooked. Researchers are working on ways to provide a single genetic screening test which can provide a complete medical diagnosis, companies are working on ways to diagnose state of health using an iPhone, etc. The approaches are invariably innovative yet may lack a fundamental scientific principle which can provide an accurate and reliable diagnostic indication. There is a need for technologies which improve the accuracy of diagnosis, overcome the limitations of current tests, and reduce the cost of diagnosing and treating disease. This article reviews one such technology: Virtual Scanning.

Virtual Scanning is based upon three observations: (i) that sense perception, and in particular colour perception, is linked to the function of the autonomic nervous system; (ii) that autonomic dysfunction influences the coherent and synchronised function of the organ networks and ultimately affects cellular and molecular biology; and (iii) the stability of the autonomic nervous system is neurally regulated. Accordingly measurements of colour perception can be used with diagnostic effect and knowledge of the structure of the autonomic nervous system and physiological systems may be used to re-establish the stability of the autonomic nervous system. In summary, the author presents a number of case studies to illustrate the potential scope for Virtual Scanning. In particular how it can be used to diagnose and treat disease.
\end{abstract}

Keywords: Virtual scanning; Autonomic nervous system

Abbreviations: ANS: Autonomic Nervous System; VS: Virtual Scanning; VSLT: Virtual Scanning Light Therapy

\section{Introduction}

There is not yet an accepted understanding of the organising principle(s) upon which the body functions, although there is recognition of higher (hyper) and lower (hypo) levels of system function and of the mechanism which has come to be known as 'homeostasis'. Perhaps the earliest recognition of the function of the central (CNS) and autonomic nervous system (ANS) came from Sherrington [1] in 1906. He concluded that the essential function of the nervous system was to coordinate or integrate the activity of various parts of the organism. Holst [2] identified that endogenous neural oscillators coordinate the rhythmic activities of systems and identified (i) the tendency of the oscillator to maintain a steady rhythm and (ii) that the oscillator influences the function of other oscillators in a manner which appears to magnetically couple it to its own frequency. Building on the work of Bernard, Cannon, von Holst, Hebb, Hess, von Bertalanffy [3], Prigogine, and others; in 1978 Sinz [4] proposed a dynamic systems theory of the organism which incorporated an understanding of regulatory mechanism, functional coordination, and oscillation or frequency. Bischof [5] summarises such progress in his review article published in 2008.

By the middle of the $20^{\text {th }}$ century research in the capitalist world had diverged from that in the communist world. Drug research became the dominant paradigm however the emphasis upon drug-based research merely highlighted the fundamental limitations of knowledge regarding how the body functions. Perhaps this was best summarised by Lander [6] when referring to the genome i.e. 'we have the parts list but we do not yet know how it works', and/or by Kandel [7] who recognised the fundamental limitations of the existing paradigm which overlooks the significance of sensory input i.e. which fails to link sensory input to cellular and molecular biology. Nevertheless there is increasingly a convergence of opinion and of research as psychologists, neurologists, neurobiologists, physiologists and others seek to explain how the body functions. The limitations of the genetic paradigm espoused by Strohman $[8,9]$ and others are becoming increasingly evident.

Meanwhile, in the communist world Anokhin [10-13] (1935/1937) had developed a theory of the functional systems as a basis for physiological integration. This was subsequently developed and refined

*Corresponding author: Graham Wilfred Ewing, Montague Healthcare, Mulberry House, 6 Vine Farm Close, Cotgrave, Nottingham, UK, Tel: 00(44)-1159890304/9899618; Fax: 00(44)-7885-755847; E-mail: graham.ewing@montaguediagnostics.co.uk; graham.ewing@montaguehealthcare.co.uk

Received April 14, 2013; Accepted April 23 2013; Published April 26, 2013

Citation: Ewing GW (2013) Virtual Scanning: A New Medical Paradigm? J Comput Sci Syst Biol 6: 093-098. doi:10.4172/jcsb.1000105

Copyright: () 2013 Ewing GW. This is an open-access article distributed under the terms of the Creative Commons Attribution License, which permits unrestricted use, distribution, and reproduction in any medium, provided the original author and source are credited. 
by Sudakov [14-21], Bekhtereva [18-22], Kryzhanovskiy [18-23], and others i.e., that disruption of the regulatory mechanism involving the nervous structures disrupts the coherent function of the organ networks i.e. physiological systems, and results in the emergence of pathological functional systems as stable pathologies. The brain adapts to assume the most stable state. It has also led to an understanding of the neural mechanisms responsible for thought, memory, emotions, and creativity; and that the body's function responds to the flow of information i.e. it is in effect an 'information technology'. Such research led to the development of Virtual Scanning (VS) technology by Grakov [24,25].

VS is a cognitive, computer-based technology which has broad diagnostic and therapeutic applicability. The test is based upon the ability to complete the computer-based test which involves recovering the colour balance of pictures presented earlier i.e., that the difference in colour perception between the presented and recovered pictures can be used diagnostically (see demonstration video on www.montaguediagnostics.co.uk ).

As a diagnostic and therapeutic modality it has been evaluated at many Russian medical clinics and is claimed [25] to be able to diagnose the onset of (all common) pathologies earlier, better, more comprehensively, safer, faster and at significantly less cost than contemporary medical technologies; and as a therapeutic modality it appears [25] able to correct the autonomic dysfunction which is manifest as many of the common morbidities. Nevertheless, although recommended for use by three Russian universities (St. Petersburg, Vladivostok and Astrakhan) following scientific evaluation, and first approved for use in the Russian Health Services in 1999/2001, where over 500 doctors have been trained to use this technology, clinical trials have not yet been conducted.

\section{Theoretical Justification}

Nobel laureate Professor Eric Kandel has highlighted 'the theoretical void between sense perception and cellular and molecular biology' [7] i.e., the stresses which we experience influence the body's function and influence the development and onset of pathologies. For example emotional stress [26-28] in the form of bereavement, loneliness, anger, frustration, grief and neglect [25] have been linked to cardiovascular problems; work-related stresses are linked to digestive problems such as oesophagitis, gastric ulcers, and the release of digestive acidity to the intestines; stress creates back spasms, asthmatic episodes, headaches, migraines, influences foetal development, suppresses immune function, constricts the arteries which lead to the heart and leads to heart attacks, etc. In the most extreme cases lack of sensory input leads to lack of neural development and ultimately to the premature onset of morbidities and earlier mortality [29].

Sensory or biological input, at or beyond the normal physiological limits [30], influences the stability of the autonomic nervous system [3133]. Psychological stress in particular influences the neural regulation of endocrine glands, the stability of the various organ networks and leads to changes at the cellular and molecular level. This autonomic instability suppresses immune function [34-36], increases susceptibility to viral infection, the incorporation of viral RNA into DNA [37], and ultimately to the onset of cellular and molecular changes which are typical of pathologies [38]. The evidence suggests that this mechanism is strongly associated with increased acidity and a reduction in the levels of magnesium which is essential for enzyme function. This leads to the inflammatory response and/or to hyper function or hypofunction in the cells. Increased acidity suppresses metabolic rate is a feature in diabetes [39], heart conditions [39], etc. This is supported by noting that psychological stress slows wound healing [40] whilst exercise, which reduces intercellular acidity, enhances the rate of wound healing [41].

Modern medicine fails to consider the mechanisms which naturally maintain the body's physiological stability and which, if understood and adapted, could be used therapeutically. Drugs and/or pharmaceutical supplements are considered to be the only solution yet for most of us who have an active lifestyle and balanced diet the body is naturally able to regulate its function and re-establish its stability and health i.e. drugs can inhibit the processes which are manifest as symptoms of pathology but it is the body's natural healing mechanism which enables the brain to re-establish homeostasis and is ultimately responsible for recovery eg. to eliminate the toxic metabolites from the oncological drugs used in chemotherapy.

The body uses the autonomic nervous system to regulate its function [42]. Sensory input and dietary/pharmacological input both influence the body's function i.e. the body is an open dynamic system which responds to both sensory input and biological input [3]. Sensory input is a biological process. It can have a positive or negative significance. All sensory input is converted into electrical and biological processes upon passage through the senses eg. in the case of visual input through the neuronal structures and opsins which exist in the retina.Intriguingly, light passes through and/or around the ganglion cells and capillary vessels in the retina before it reaches the rods and cones. Extremes of sensory input influence neural synchronisation and the stability of system function [43] eg. leading to increased blood pressure. Drugs also have the same effect e.g. caffeine and nicotine raise EEG frequencies. Similarly altering the prevailing EEG frequencies using biofeedback techniques alters physiological stability i.e. the spectrum of metabolites. The autonomic nervous system responds to sensory input of which visual input is the most dominant [44] and physiologically significant. Alterations to the body's stability influence colour perception and can be influenced by colour e.g. red influences appetite, stimulates aggression, and is a sexual stimulant. By contrast a pastel shade of green is often used in heart wards where it is recognised that it can slow the beat of the heart. Light and colour influence the body's function i.e. over 100 common medical conditions are known to respond to the therapeutic effect of natural sunlight. Light and colour are essential photostimulants which activate proteins and related biological systems.

The link between sensory input and the ANS is recognised although the mechanism has not yet been established beyond reasonable doubt. As stated by geneticist Professor Eric Lander of MIT when referring to the genome 'we now know the parts list but we do not yet know how it works'.

As the body's function is wholly biochemical it follows that any changes to sense perception must have a biological basis. The greater the intensity or significance of the multi-sensory event the greater will be its effect upon cellular morphology and ultimately upon molecular biology. That disease and drugs both influence colour perception indicates that the likely solution lies in understanding the link between colour perception and biological change; in particular, the properties of the most basic biological components, proteins, and the structure and function of the autonomic nervous system.

\section{Diagnostic}

Proteins are visually active. They absorb and emit light. Alterations 
to the levels of proteins and protein reactivity i.e. genotype and phenotype, influence the spectrum of light which is absorbed and, in particular, which is emitted [45]. For example (i) diabetes is a problem associated with the reduced level of the key protein insulin (genotype) and the lessened ability of insulin to react and/or perform its cellular function (phenotype); (ii) the emergence of diabetes is accompanied by changes to colour perception (n.b. glycated haemoglobin and other glycated proteins are highly bioluminescent). Originally this change of colour perception was considered to be associated with the onset of diabetic retinopathy however articles have been published which indicate that such changes to colour perception occur before the onset of diabetic retinopathy [46-48] i.e., at the onset of the pathology. Accordingly changes to colour perception are directly related to the onset of pathologies. Every pathological reaction releases a biophoton of particular colour and intensity. When this bioluminescence reaches a threshold level, considered to be typically 70 biophotons per second, it starts to influence colour perception i.e. the eye detects changes from typically 70 biophotons per second up to 10 to the power of 9 biophotons per second. The eye performs the function of a spectrophotometric detector of immense sensitivity. The currently considered mechanism is that this bioluminescence is released from blood in the choroid and penetrates the retina via Bruch's membrane and capillary blood vessels. A number of precedents (not listed) support this argument. A similar effect is noted in the auditory spectrum i.e. pathologies influence the auditory spectrum. Pathologies also influence smell, taste and touch.

A biomathematical model is used to interpret the sensory data which has been derived from the cognitive VS test which is conducted on a computer. This is analogous to other diagnostic technologies which use electromagnetic radiation and mathematics to interpret the data. Virtual Scanning has been evaluated by doctors and assessed to be $100 / 82.4 \%$ accurate [25] i.e., circa $20 \%$ more accurate than contemporary methods of diagnosing disease.

Instead of determining the genetic spectrum the technique effectively by-passes the genes and focusses solely upon the light emitting properties of proteins [49]. By comparison genetic screening can identify genetic conditions in the foetus and in young children whereas Virtual Scanning is applicable to those who can use the technology i.e., typically age 7 years until the onset of cognitive decline.

Virtual Scanning may be a technology platform as powerful as genetic screening, if not more so, because all diseases are influenced by both genotype and phenotype and both can be quantified using VS. For instance (i) in the BRCA gene which is recognised to cause breast cancer it is recognised that an environmental trigger acts to stimulate the cancerous process; (ii) diabetes is a combination of genetic (type 1) and phenotypic (type 2) disorder. In recent articles we have written about the significance and relationship between genotype and phenotype. As stated in the literature accompanying this article VS does not look at the genes but instead looks at the properties of proteins which are genetically expressed. This appears to be a better concept than genetic screening. There is certainly a role for genetic screening but at current estimates of $£ 1000$ per test this is hugely expensive as a general screening modality. By comparison, VS will be priced at typically $£ 5-50$ per test depending upon the nature of the pathology(s) to be identified.

Virtual Scanning is able to diagnose every medical condition (see Example Report Appendix 1). The only limitation is whether the algorithm has been generated for the condition(s). Currently the technique diagnoses the common pathologies which are associated with each organ. It is possible to extend this technique and develop algorithms for each genetic condition e.g.in genetic conditions which affect typically 1 in 10,000. VS has identified a case of 'mesenteric vascular insufficiency' [50] which affects 1 in 250,000. It was able to diagnose mesenteric insufficiency because it is a complex multisystemic condition. In such cases no single protein, gene, combination of genes is able to accurately diagnose the condition because of the multi-systemic and multi-biological nature of the condition however such complex conditions can be diagnosed by looking at the pattern of pathologies which develop e.g. (i) in cystic fibrosis this can be expected to show a pattern of lung disorders and a pattern of disorders in other organs and (ii) migraines [51] are caused by the combined function of the different pathologies which impede the flow of oxygen to the brain e.g. heart function, low blood pressure, blood viscosity, patent foramen ovale, spondylosis, and vertebral artery syndrome.

(Each signal denoted in the example report is a mathematical measure of genotype and phenotype for each pathology. The blue signals denoted as compensatory refer to genotype, and the red signals denoted as pathology refer to phenotype. Signals above 10 units are at the presymptomatic level whilst signals below 10 units are at the presymptomatic level. For example in the case of diabetes mellitus there will be a genotype signal indicative of the lessened amount of insulin (type 1) and a phenotype signal indicative of insulin-resistance (type 2). The greater the signals the more severe are the morbidity).

The one remaining issue is to link the ANS to cellular and molecular biology, however perhaps this is already quite self-evident. If it is cellular it must involve the organs in which the cells reside and the organ networks in which the organs function. If so, the autonomic nervous system and organ networks are synonymous.

This explains how the body's function can be influenced by sensory input and biological factors. It confirms that the human organismis an open and dynamic system ${ }^{3}$ which is influenced by sensory input and by biological input. It also indicates that the function of the brain is linked to issues which, directly or indirectly, regulate the body's function and lead us to pursue measures which solve the basic physiological requirements e.g. for food, drink, warmth, family, security, etc.

VS is able to diagnose the health of people who are colour blind. Such people have a lack of one or more cone subtypes. They are not blind to particular colours but experience an inability to distinguish between the two groups of colours which can be distinguished by people with normal colour vision.

\section{Example case studies}

1. A lady with a duodenal discomfort following unsatisfactory results from her GP who advised that she did not have a problem. VS detected a problem with the duodenum, probably a duodenal ulcer, and referred her to her GP who under duress carried out a further consultation and again gave a negative report. She reported her dissatisfaction with the VS result yet she was admitted to hospital with blood discharges in her stools (the classic 'tarry stool') just one week later and spent over one week in hospital being treated for a 'perforated' duodenal ulcer. Upon discharge from hospital she confirmed the accuracy of the VS report. The lady gained a compensatory settlement as a result of the misdiagnosis by the GP.

2. A practitioner working with VS, at the introductory and investigative stages of her work, noted the steady reduction of cell count in her blood as indicated by VS. As she had previously 
been treated for leukaemia, and her most recent test had shown a steady level of leukocytes, she discounted the possibility of reoccurrence of her lymphoblastic leukaemia. A subsequent medical check identified the reoccurrence of leukaemia.

3. A male of 60 years, problems with a stress-related 'irritable bowel' condition influencing his digestion and elimination for many years which has been especially severe during 2011/12. Indications: gastric reflux, severe bloating/wind and severe diarrhoea, typically 90 minutes after a meal. Patient used milk of magnesia which had some effect therefore gastric acidity was playing a role in the problem. Omeprazole calmed the symptoms but not solve the problem. His doctor was unable to advise anything other than an endoscopic examination which was declined. Blood test did not indicate the presence of helicobacter pylorii. VS indicated symptomatic levels of oesophagitis (gastric reflux); stomach ulcer; duodenal ulcer; and sigmoiditis and colitis in the intestines.

4. A lady of circa 40 years, problems of lack of energy. VS diagnosed the early onset of pancreatic cancer from which the patient died several years later.

5. A lady of 68 years with breathing and coughing fits. VS diagnosed bronchiectactic disease several months before it could be confirmed by conventional diagnostic procedures at her local hospital. After several months of VSLT the breathing and coughing fits no longer occurred.

6. A lady of 59 years, who had experienced regular migraine type events from the age of 11 until 59 years: the most recent resulting in her being hospitalised in a semi-conscious state. Various medications were prescribed but none had any effect. She was taken home in an ambulance, and advised to sit in a darkened room until the migraine subsided. A VS assessment indicated migraine and epilepsy, impaired cerebral circulation, impaired spinal circulation as a result of vertebral artery syndrome, cardiosclerosis, osteochondrosis and idiopathic hypotension.

\section{Therapeutic}

That light can also be used therapeutically is also significant. Light has been used therapeutically for thousands of years. It is currently used in complementary and alternative medicine, laser therapy, syntonic optometry and as a biofeedback type therapy to treat dyslexia, migraine, etc. Of particular interest is that flashing lights can cause photosensitive events but can also be used to treat photosensitivity. This illustrates that there is a scientific principle involved i.e. that the appropriate selection of colour/light and frequency can be deployed with therapeutic effect to re-establish the stability of the autonomic nervous system i.e. to photostimulate specific biological processes. Such principle, of neural synchronisation, biofeedback or brain-wave coherence, has been the subject of speculation for many years by eminent researchers e.g. to explain the circadian rhythms [52] and/or cycles which influence the release of insulin, the day/night cycle, the menstrual cycle, etc.

Neural networks fire in a coherent manner. If so, the neural networks must perform a physiologically significant purpose. It is likely that the greater the extent of the neural coherence the lower will be the neural (EEG) frequency and the greater will be its physiological and psychological effect.

Initial indications are that Virtual Scanning Light Therapy (VSLT) is able to treat a wide range of ailments [25]. VSLT has been used to successfully treat a range of disorders including sleep disorders [53], breathing disorders, migraine w/o aura [54], circulatory problems, developmental dyslexia $[55,56]$, etc. Our experience largely supports the claims by Vysochin, in an unpublished article prepared for the Russian parliament THE DUMA [25], that the technique is circa 93\% effective. The technique works effectively to treat autonomic dysfunction. It appears likely to be typically $75-85 \%$ effective. By comparison $90 \%$ of drugs are considered to be ineffective in $50 \%$ of those treated [57].

\section{Example case studies}

1. A man of 78 years was dissatisfied with his GP's diagnosis when he consulted him with type 2 diabetes, and a swollen foot. $\mathrm{He}$ became disillusioned with the diagnosis when prescribed antibiotics-presumably as a preventative measure against infection. His mobility and quality of life was at a very low level. Over a 6 months period, during which he undertook a course of VSLT, his swollen foot ceased to be swollen, his blood sugar levels stabilised. He recovered his mobility, improved circulation, improved energy, and completely normalised quality of life.

2. A man of 65 years suffering from severe Depression, who had been treated over many years with many different forms of medication, which, he advised, never had any effect on his health. Following a course of VSLT he has been completely free of depression.

3. A man of 76 years with Prostate cancer and Mesothelioma. Following chemotherapy and radiotherapy he had severely restricted breathing due to the accumulation of fluid on his lungs which required surgical intervention in the form of a stent but which failed to cure the problem of accumulating fluid. He required assistance to walk. The medical prognosis was for only short-term survival. After a period of 2-3 months VSLT the patient no longer had any fluid on the lungs to the astonishment of his consultant. Medical Scanning techniques (x-rays) showed the complete absence of fluid on his lungs. After 3-4 months of VSLT his weight had increased by $5 \mathrm{kgs}$, he walked unaided although slowly, his breathing was dramatically improved, his eyes alert, his mental clarity was much improved and he was happy. He died 1-2 years later i.e., 1-2 years later than forecast by his consultant.

4. A lady of 56 years suffering from the after effects of a head trauma. Her health was of regular migraines and of taking anti-epilepsy medications. She was very fragile, had the appearance of being sedated, and was depressed by the effect that her condition was having upon the quality of her life and of the lives of her immediate family. She received VSLT for 3-4 months. After 2 modules of therapy, she no longer needed her medication and recovered a happy and healthy demeanor. She commented upon feeling calmer and more relaxed.

5. A lady of circa 40 years with Trigeminal Neuralgia has been seeking a cure for her medical condition for many years. Conventional methods of treating this condition had been largely ineffective. To this end she has travelled widely: in her words 'she has lost count of the money spent seeking a cure'. After one week of VSLT she advised that it had drastically reduced the level of her pain. 
6. A male of 59 years with Sleep Apnoea. The abnormal sleep episodes were affecting his ability to drive and the quality of his private life. Oxygen therapy was being used to reduce the severity of the symptoms. He was offered surgery to improve his breathing which was hoped would mitigate the condition. He declined the surgery and undertook several months of VSLT. After c6 months his consultant advised that he was no longer suffering from the condition. He wrote: 'I just had to write and tell you how much better I feel after using your treatment therapy. I recently went to the hospital for my annual Sleep Apnoea appointment and the professor told me that I no longer had the condition. He asked how this could be and I told him of your therapy. To say he was stunned would be an understatement. My general wellbeing has also improved. Thank you once again'.

7. A lady of $\mathrm{c} 21$ years was fainting up to 5 times per day (Syncope). Medical tests were inconclusive. She had been steadily losing weight for several months. Virtual Scanning identified that her problem was due to low blood volume and anaemia. Within one month of commencing VSLT the fainting episodes ceased.

8. A male of c65 years had been unable to speak for almost 5 years. He could only speak in the most guttural manner which was largely incomprehensible. He had been advised that his condition dysarthria had been caused by a mild stroke. Despite exhaustive testing and therapies nothing was able to solve the problem. After 6 days of VSLT he had completely recovered his ability to speak clearly and intelligibly.

9. A lady of c60 years spent 2-3 years without sleep following a brain operation to remove a cancerous tumour. Her condition was emotionally unstable. Within 2 weeks of receiving her first VSLT she had settled into regular sleeping for typically 12-14 hours each day. Her health steadily improved, in addition to her emotional stability, and general wellbeing.

10. A girl of 10 years with developmental dyslexia had been assessed as having a reading age of 8 years i.e. 2 years below her calendar age. After 6 months of VSLT her reading age was reassessed as that of a 14 y.o. i.e. 3 years in advance of her calendar age. Her writing has improved although there are indications of errors in spelling. Her mother considers that the use of spellcheck and computers will largely overcome this in future. Her first set of examination results at a new private school were 9 grades 1 and 2 (above $70 \%$ ) and the remaining two examinations as grade 3 (60-70\%).

11. A lady of 59 years (see section 3 diagnostic case study (vi)). Since starting VSLT she has not had any further migraine symptoms. A further effect of this therapy is worth noting. Several years previously this lady had required a single mastectomy to remove indications of breast cancer. This involved removal of the lymph nodes which resulted in poor drainage and hence swelling of her arm. After 4 months VSLT the lady returned to her oncologist for her annual check who was astonished to note that the lady had little, if any, remaining swelling in her arm.

\section{Conclusion}

The principles, upon which Virtual Scanning was developed, originated from laser research conducted by Dr Igor Gennadyevich Grakov at the University of Novosibirsk in the early 1980's. Virtual
Scanning is based upon an understanding of the link between sense perception, the autonomic nervous system, physiological systems, and cellular and molecular biology. It fills the theoretical void [7] identified by Kandel.

In addition the understanding of the relationship between sense perception, the autonomic nervous system, and physiological systems may lead to better understanding of Ayurveda and many Complementary and Alternative Medical techniques [58] which are based upon sensory input and the artificial stimulation of system function.

Virtual Scanning was first approved for use by the Russian Health Services in the period 1999/2001. Over 550 doctors have been trained to use this technique. Nevertheless the project has encountered financial, political and regulatory issues which require that investment is raised to fund programmes of clinical studies, regulatory approval, marketing programmes, etc.

\section{References}

1. Sherrington CS (1906) The Integrative Action of the Nervous System. Charles Scribner and Sons, NY, USA.

2. Von Holst W (1939) Die Relative Koordinationals Phanomen und als Methode Zentral nervoser Funktions analyse. Ergebn Physiol 42: 228-306.

3. Bertalanffy $L$ von (1967) General theory of systems application to physiology Social Sci Inform Sci Socials 6: 126.

4. Sinz R (1978) Zeitstrukturen und Organismische Regulation. Akademie Verlag, Berlin.

5. Bischof M (2008) Synchronization and Coherence as an Organizing Principle in the Organism, Social Interaction, and Consciousness. NeuroQuantology 6 : $1-12$.

6. http://www.pbs.org/wgbh/nova/genome/deco_lander.html

7. Kandel E (2006) The New Science of Mind. Scientific American Mind 17: 62 69.

8. http://www.psrast.org/strohmnewgen.htm

9. Strohman RC (1997) The coming Kuhnian revolution in Biology. Nature Biology 15: $194-200$

10. Anokin PK (1935) The problem of the center in the physiology of nervous activity. Gorky 9: 70.

11. Anokhin PK (1949) The functional system as the basis for the integration of nervous process during embryogenesis. Proceedings of the All-Union Conference of Physiologists, Biochemists and Pharmacologists, Tbilisi: 148156

12. Anokhin PK (1949) Reflex and functional system as factors of physiological integration. Fiziol Zh SSSR Im I M Sechenova 35: 491-503.

13. Anokhin PK (1974) Biology and Neurophysiology of the Conditioned Reflex and its Role in Adaptive Behavior. Pergamon Press, NY, USA.

14. Sudakov KV, Volkova KA (1978) Prospects for the development of the functional system theory. Usp Fiziol Nauk 9: 3-27.

15. Sudakov KV (1982) New aspects of the Theory of a Functional System. Vestn Akad Med Nauk SSSR 2: 3-14.

16. Sudakov KV (1982) Functional System Theory in Physiology. Agressologie 23 167-176.

17. Sudakov KV (1988) Gene determination of Physiological Functions: Experimental Approaches based on the Functional Systems Theory. Fiziol Zh SSSR Im I M Sechenova 74: 8-16.

18. Kryzhanovskil̆ GN, Adrianov OS, Bekhtereva NP, Negovskiĭ VA, Sudakov KV, et al. (1995) Integrative Activity of the Nervous System in Health and in Disease. Vestn Ross Akad Med Nauk 8: 32-36.

19. Sudakov KV, Viktorov VA, lumatov EA (1999) New medical technologies in 
human health evaluation on the basis of functional systems theory. Vestn Ross Akad Med Nauk 9: 19-22.

20. Sudakov KV (2011) From genes to Functional Systems. Patol Fiziol Eksp Ter 4: 3-6.

21. Sudakov KV (2011) The Information Theory of Brain Systemic Activity. Vestn RossAkad Med Nauk 11: 3-12.

22. Bekhtereva NP, Bundzen PV, Gogolitsyn YuL, Ilyukhina VA, Medvedev SV (1980) Physiological correlates of states and activity in the central nervous system. Hum Physiol 6: 367-380.

23. Kryzhanovskii GN (2004) Some categories of general pathology and biology: health, disease, homeostasis, sanogenesis, adaptation, immunity New approaches and notions. Pathophysiology 11: 135-138.

24. Grakov IG (1985) Strannik Diagnostic and Treatment System: a Virtual Scanner for the Health Service. Minutes of Meeting No. 11 of the Praesidium of the Siberian of the Academy of Medical Sciences of the USSR (AMN) held in Novosibirsk 4 December.

25. Vysochin Yu (2001) Methodology and Technology of Invigoration of Different Population Orders. Consolidated 5 year Research Plan of Physical Training, Sports and Tourism State Committee of the Russian Federation.

26. Sudakov KV (1979) Pathogenesis of disorders of heart activity under emotional stress. Patol Fiziol Eksp Ter 3: 16-22.

27. Kiecolt-Glaser JK, Glaser R (2002) Depression and immune function: Central pathways to morbidity and mortality. J Psychosom Res 53: 873-876.

28. Mostofsky E, Maclure M, Sherwood JB, Tofler GH, Muller JE, et al. (2012) Risk of acute myocardial infarction after the death of a significant person in one's life: the Determinants of Myocardial Infarction Onset Study. Circulation 125: 491-496.

29. Perry BD (2002) Childhood Experience and the Expression of Genetic Potential: What Childhood Neglect tells us about Nature and Nurture. Brain and Mind 3: 79-100.

30. Kiecolt-Glaser J, Glaser R (1988) Major life changes, chronic stress, and immunity. Adv Biochem Psychopharmacol 44: 217-224.

31. Cacioppo JT, Berntson GG, Malarkey WB, Kiecolt-Glaser JK, Sheridan JF, et al. (1998) Autonomic, neuroendocrine, and immune responses to psychological stress: the reactivity hypothesis. Ann N Y Acad Sci. 840: 664-673.

32. Kiecolt-Glaser JK, Glaser R, Cacioppo JT, Malarkey WB (1998) Marital stress: immunologic, neuroendocrine, and autonomic correlates. Ann N Y Acad Sci 840: 656-663.

33. Malarkey WB, Kiecolt-Glaser JK, Pearl D, Glaser R (1994) Hostile behavior during marital conflict alters pituitary and adrenal hormones. Psychosom Med 56: 41-51.

34. Glaser R, Kiecolt-Glaser JK (1986) Stress and immune function. Clinica Neuropharmacology Suppl 4: 485-487.

35. Glaser R, Rice J, Speicher CE, Stout JC, Kiecolt-Glaser JK (1986) Stress depresses interferon production by leukocytes concomitant with a decrease in natural killer cell activity. Behav Neurosci 100: 675-678.

36. Glaser R, Kiecolt-Glaser JK, Speicher CE, Holliday JE (1985) Stress, loneliness, and changes in herpesvirus latency. J Behav Med 8: 249-260.

37. Glaser R, Thorn BE, Tarr KL, Kiecolt-Glaser JK, D'Ambrosio SM (1985) Effects of stress on methyltransferase synthesis: an important DNA repair enzyme. Health Psychol 4: 403-412.

38. Sgoutas-Emch SA, Cacioppo JT, Uchino BN, Malarkey W, Pearl D, et al. (1994) The effects of an acute psychological stressor on cardiovascular, endocrine, and cellular immune response: a prospective study of individuals high and low in heart rate reactivity. Psychophysiology 31: 264-271.

39. Ewing GW (2012) The Regulation of pH is a Physiological System. Increased Acidity alters Protein Conformation and Cell Morphology and is a Significant Factor in the onset of Diabetes and other common pathologies. The Open Systems Biology Journal 5: 1-12.
40. Kiecolt-Glaser JK, Marucha PT, Malarkey WB, Mercado AM, Glaser R (1995) Slowing of wound healing by psychological stress. Lancet 346: 1194-1196.

41. Emery CF, Kiecolt-Glaser JK, Glaser R, Malarkey WB, Frid DJ (2005) Exercise accelerates wound healing among healthy older adults: a preliminary investigation. J Gerontol A Biol Sci Med Sci 60: 1432-1436.

42. Ewing GW, Ewing EN (2008) Cognition, the Autonomic Nervous System and the Physiological Systems. Biogenic Amines 22: 140-163.

43. Ewing GW (2010) Mathematical Modeling the Neuroregulation of Blood Pressure using a Cognitive Top-down Approach. N Am J Med Sci 2: 341-352.

44. Ewing GW, Parvez SH, Grakov IG (2011) Further Observations on Visual Perception: the influence of pathologies upon the absorption of light and emission of bioluminescence. The Open Systems Biology Journal 4: 1-7.

45. Ewing GW, Parvez SH (2010) The Multi-systemic Nature of Diabetes Mellitus: genotype or phenotype?. N Am J Med Sci 2: 444-456.

46. Kurtenbach A, Schiefer U, Neu A, Zrenner E (1999) Preretinopic changes in the colour vision of juvenile diabetics. $\mathrm{Br} \mathrm{J}$ Ophthalmol 83: 43-46.

47. Beisswenger PJ, Makita Z, Curphey TJ, Moore LL, Jean S, et al. (1995) Formation of immunochemical advanced glycosylation end products precedes and correlates with early manifestations of renal and retinal disease in diabetes. Diabetes 44: 824-829.

48. Ismail GM, Whitaker D (1998) Early Detection of Changes in Visual Function in Diabetes Mellitus. Ophthalmic Physiol Opt 18: 3-12.

49. Ewing GW (2013) A Comparison of the Diagnostic Scope of Biomarke techniques, Genetic Screening and Virtual Scanning. Immunol Endocr Metab Agents Med Chem 13: 35-45

50. Stemmer EA, Connolly JE (1973) Mesenteric Vascular Insufficiency. Identification and Management. Calif Med 118: 18-29.

51. Ewing GW, Ewing EN, Parvez SH (2009) The Multi-systemic Origins of Migraine. Biogenic Amines 23: 1-52.

52. Shannahoff-Khalsa DS, Kennedy B, Yates FE, Ziegler MG (1996) Ultradian rhythms of autonomic, cardiovascular, and neuroendocrine systems are related in humans. Am J Physiol 270(4 Pt 2): R873-R887.

53. Ewing GW, Nwose EU, Ewing EN (2009) Obstructive Sleep Apnea Management with Interactive Computer Technology and Nutrition: Two Case Reports. J Altern Complement Med 15: 1379-1381.

54. Ewing E, Ewing G, Nwose EU (2009) Migraine can be managed with Virtua Scanning: case report. The Open Complementary Medicine Journal 1: 16-18.

55. Ewing GW, Ewing EN, Parvez SH (2009) Developmental Dyslexia: the link with the Autonomic Nervous System and the Physiological Systems. Biogenic Amines 23: 115-190.

56. Ewing GW (2009) A Theoretical Framework for Photosensitivity: Evidence of Systemic Regulation. J Comput Sci Syst Biol 2: 287- 297

57. Spear BB, Heath-Chiozzi M, Huff J (2001) Clinical application of pharmacogenetics. Trends Mol Med 7: 201-204.

58. Ewing GW (2009) Does an improved understanding of the nature and structure of the Physiological Systems lead to a better understanding of the therapeutic scope of Complementary \& Conventional Medicine? J Comput Sci Syst Bio 2: 174-179. 